\title{
Caractérisation anatomique de la carcasse de la canette de barbarie (Cairina moschata L.)
}

\author{
F.H. RICARD \\ avec la collaboration technique de G. MARCHE \\ INRA, Station de Recherches avicoles \\ Centre de Recherches de Tours \\ Nouzilly, F-37380 Monnaie
}

\begin{abstract}
Résumé
Un lot de 22 canards de barbarie de sexe femelle a été abattu à l'âge de 10 semaines en vue de préciser l'importance des différents éléments de la carcasse, en particulier les dépôts gras et la peau, et d'estimer les corrélations qui relient ces éléments.

Pour l'échantillon étudié, la poitrine sans os représente 16,2 p. 100 du poids vif, les ailes 9,7 p. 100 , l'ensemble cuisses + pilons 17,5 p. 100 , le dépôt gras abdominal 4,1 p. 100 , les dépôts gras sous-cutanés de la carcasse 4,0 p. 100 et la peau disséquée sur la carcasse (sans les ailes ni le croupion) 10,7 p. 100 . Les corrélations entre poids bruts des différentes parties de la carcasse sont généralement élevées; celles entre le poids vif et les pourcentages des dépôts gras sont de l'ordre de 0,6 . Les corrélations entre pourcentages font apparaître une relative indépendance entre le développement de la peau et celui du tissu gras.

Les résultats ont été comparés avec ceux de trois autres expérimentations portant également sur des canettes de barbarie âgées de 10 semaines. On aboutit à des valeurs du même ordre de grandeur. Comme chez le poulet, l'ensemble cuisses + pilons de la canette de barbarie donne une bonne image du pourcentagge de peau et de gras sous-cutané de la carcasse entière.
\end{abstract}

Nos résultats peuvent intéresser les abattoirs qui souhaitent développer la découpe des canettes de barbarie.

Mots clés : Canard de barbarie, parties de carcasse, gras abdominal, gras sous-cutané, peau, corrélations.

\section{Introduction}

Après avoir pris, en France, une place prépondérante comme fournisseur de carcasses de palmipèdes à rôtir, le canard de barbarie (Cairina moschata L.) voit sa production continuer à augmenter grâce au développement de la découpe et de produits élaborés. Dans ces conditions, les facteurs de variation de la composition anatomique et de l'engraissement de cette espèce prennent une importance de plus en plus grande.

De nombreux auteurs ont étudié la composition anatomique et/ou chimique de la carcasse du canard de barbarie, en particulier en fonction de l'âge d'abattage et souvent 
en comparaison avec le canard commun. Parmi les travaux récents, on peut citer ceux de Romboli (1980), Pingel \& Schneider (1981), Karasinski et al. (1981), Seemann (1983), Ziegler et al. (1985), Torges (1986) et de Ricard (1986). Les travaux de Babile et al. (1986) fournissent des éléments concernant la découpe des carcasses telle qu'on la pratique dans les abattoirs artisanaux du Sud-Ouest de la France pour les palmipèdes gras.

Il nous a semblé intéressant d'étudier en détail la répartition des différentes parties de la carcasse du canard femelle ainsi que des éléments de la peau et du dépôt gras sous-cutané. D'une part, la connaissance de cette répartition est utile pour les abattoirs qui voudraient promouvoir la canette de barbarie grâce à la découpe. D'autre part, nous voulions voir si la dissection des cuisses et des pilons pouvait fournir une bonne estimation du pourcentage total de la peau et du tissu gras sous-cutané dans la carcasse entière, comme c'est le cas chez le poulet (RiCARD, 1984). Ce travail a été réalisé sur un lot de 22 canettes de barbarie abattues à l'âge de 10 semaines. Les résultats sont présentés ici et discutés en comparaison avec ceux d'autres chercheurs.

\section{Matériel et méthodes}

Les animaux utilisés appartenaient à un croisement commercialisé par la Société Grimaud Frères. Ils ont été élevés en cages collectives au printemps 1984, dans des conditions analogues à celles décrites précédemment (RICARD, 1986).

A l'âge de 10 semaines, un échantillon de 22 femelles, représentatif de l'ensemble du lot expérimental, a été pesé après un jeûne de 16 heures puis abattu. La plumaison était dégrossie à l'aide d'une plumeuse à disques puis finie à la cire. Au cours de l'éviscération, on a pesé les dépôt gras abdominaux (situés contre la paroi abdominale et autour du gésier et du ventricule succenturié), l'ensemble des abats consommables (gésier musculaire + cœur + foie sans la véscicule biliaire) ainsi que le cou. La totalité des autres viscères a été enlevée pour obtenir le poids de la carcasse éviscérée.

Sur cette dernière, on a prélevé et pesé le croupion (avec la glande uropygienne), les ailes, l'ensemble cuisses + pilons ainsi que la poitrine (totalité des muscles pectoraux avec la peau qui les recouvre). La poitrine a ensuite été disséquée pour séparer la peau, le dépôt gras sous-cutané, le muscle grand pectoral (pectoralis superficialis) et les muscles petits pectoraux (pectoralis profundus, selon la nomenclature de CHAmberLaIN, 1943). Sur l'ensemble cuisses + pilons ainsi que sur l'ensemble reste de carcasse + cou, on a séparé et pesé la peau et le dépôt gras sous-cutané. Enfin, on a calculé le poids total de la peau et des dépôts gras sous-cutanés qui ont été disséqués.

Les mesures de rendement ont été obtenus en calculant les pourcentages du poids de chacun des éléments définis ci-dessus, d'une part par rapport au poids vif, d'autre part par rapport à chaque partie de la carcasse d'où ils provenaient.

On a ensuite calculé les paramètres statistiques (moyenne et écart-type) pour toutes les variables ainsi que les corrélations phénotypiques, d'une part entre les poids bruts, d'autre part entre les variables exprimées en pourcentages. 


\section{Tableau 1}

Moyenne et écart-type (entre parenthèses) des composants anatomiques de la carcasse de canards de barbarie femelles âgées de 10 semaines $(N=22)$, exprimés soit en poids bruts, soit en pourcentages du poids vif.

Means and standard deviations of anatomical carcass components of 10-week-old female muscovy ducklings expressed as raw weight or as percent of live weight.

\begin{tabular}{|c|c|c|c|c|c|}
\hline \multirow{2}{*}{$\begin{array}{c}\begin{array}{c}\mathbf{n}^{\circ} \\
\text { variable }\end{array} \\
1\end{array}$} & \multirow{2}{*}{\begin{tabular}{c}
\multicolumn{1}{c}{$\begin{array}{c}\text { Partie anatomique } \\
\text { Anatomical carcass } \\
\text { components }\end{array}$} \\
$\begin{array}{l}\text { Poids vif avant abattage } \\
\text { (live weight before slaughter) }\end{array}$
\end{tabular}} & \multicolumn{2}{|c|}{$\begin{array}{l}\text { Poids bruts (g) } \\
\text { Raw weight }\end{array}$} & \multicolumn{2}{|c|}{$\begin{array}{c}\text { Pourcentages } \\
\text { du poids vif } \\
\text { Percent of live weight }\end{array}$} \\
\hline & & 2123,0 & $(152,8)$ & & \\
\hline 2 & $\begin{array}{l}\text { Dépôt gras abdominal } \\
\text { (abdominal fat deposits) }\end{array}$ & 87,6 & $(26,3)$ & 4,1 & $(1,02)$ \\
\hline 3 & $\begin{array}{l}\text { Abats consommables }{ }^{(1)} \ldots \ldots \ldots \\
\text { (edible offals : gizzard }+ \text { heart }+ \text { liver) }\end{array}$ & 80,1 & $(7,3)$ & 3,8 & $(0,35)$ \\
\hline 4 & $\underset{(\text { neck })}{\operatorname{Cou}} \ldots \ldots \ldots \ldots \ldots \ldots \ldots$ & 80,2 & $(5,2)$ & 3,8 & $(0,27)$ \\
\hline 5 & $\begin{array}{l}\text { Carcasse éviscérée } \ldots \ldots \ldots \ldots \ldots \\
\text { (eviscerated carcass) }\end{array}$ & 1303,5 & $(96,2)$ & 61,4 & $(1,49)$ \\
\hline 6 & $\begin{array}{l}\text { Poitrine totale sans os } \ldots \ldots \ldots \\
\text { (total breast without bone) }\end{array}$ & 344,6 & $(30,8)$ & 16,2 & $(0,61)$ \\
\hline 7 & Dont $: \underset{\text { (breast skin) }}{\text { Peau } \ldots \ldots \ldots \ldots}$ & 75,2 & $(12,0)$ & 3,5 & $(0,37)$ \\
\hline 8 & $\underset{\text { (sub-cutaneous fat })}{\text { Gras sous-cutané }} \ldots \ldots$ & 8,1 & $(2,9)$ & 0,4 & $(0,12)$ \\
\hline 9 & $\begin{array}{l}\text { Muscle grand pectoral } \\
\text { (pectoralis major muscle) }\end{array}$ & 227,8 & $(17,4)$ & 10,7 & $(0,47)$ \\
\hline 10 & $\begin{array}{l}\text { Muscles petits pectoraux } \\
\text { (pectoralis minor muscles) }\end{array}$ & 31,6 & $(2,2)$ & 1,5 & $(0,10)$ \\
\hline 11 & $\underset{\text { (wings) }}{\text { Ailes }} \ldots \ldots \ldots \ldots \ldots \ldots \ldots$ & 205,5 & $(13,3)$ & 9,7 & $(0,50)$ \\
\hline 12 & $\begin{array}{l}\text { Cuisses }+ \text { pilons } \ldots \ldots \ldots \ldots \ldots \\
\text { (thighs }+ \text { drumsticks })\end{array}$ & 370,6 & $(30,2)$ & 17,5 & $(0,89)$ \\
\hline 13 & Dont : $\begin{aligned} & \text { Peau } \\
& \left(T+D_{\text {skin }}\right)\end{aligned}$ & 65,3 & $(7,9)$ & 3,1 & $(0,29)$ \\
\hline 14 & $\begin{array}{l}\text { Gras sous-cutané } \ldots \\
(T+D \text { subcutaneous fat })\end{array}$ & 44,9 & $(12,2)$ & 2,1 & $(0,52)$ \\
\hline 15 & $\begin{array}{l}\text { Croupion } \ldots \ldots \ldots \ldots \\
\text { (tail with preen gland) }\end{array}$ & 63,7 & $(7,7)$ & 3,0 & $(0,33)$ \\
\hline 16 & $\begin{array}{l}\text { Reste de carcasse }+ \text { cou } \ldots \ldots \\
\text { (remaining part with neck) }\end{array}$ & 396,2 & $(35,7)$ & 18,7 & $(1,02)$ \\
\hline 17 & Dont : $\underset{\text { (remaining skin) }}{\text { Peau } \ldots \ldots \ldots}$ & 87,7 & $(11,2)$ & 4,1 & $(0,37)$ \\
\hline 18 & $\begin{array}{l}\text { Gras sous-cutané } \\
\text { (subcutaneous fat) }\end{array} \ldots \ldots$ & 32,4 & $(6,8)$ & 1,5 & $(0,25)$ \\
\hline 19 & $\begin{array}{l}\text { Total peau disséquée }{ }^{(2)} \ldots \ldots \ldots \\
\text { (total dissected skin) }\end{array}$ & 228,2 & $(27,2)$ & 10,7 & $(0,75)$ \\
\hline 20 & $\begin{array}{l}\text { Total gras sous-cutané }{ }^{(2)} \\
\text { (total subcutaneous fat) }\end{array}$ & 85,5 & $(19,9)$ & 4,0 & $(0,71)$ \\
\hline
\end{tabular}

(1) Abats = gésier musculaire + cœur + foic sans vésicule biliaire, pesés globalement. Offals = gizzard + heart + liver without gall-bladder, weighed together.

(2) Carcasse totale à l'exception de la tête, des ailes et du croupion mais avec le cou. Total carcass except head, wings and tail but with neck. 


\section{Résultats}

Les paramètres statistiques (moyenne et écart-type) du poids vif et du poids de chaque élément de la carcasse sont indiqués dans les tableaux 1 et 2 . La poitrine sans os, qui est la partie de la carcasse ayant la plus forte valeur marchande représente, dans notre échantillon, un peu plus de $16 \mathrm{p}$. 100 du poids vif, dont 4 p. 100 pour la peau et le gras sous-cutané et 11 p. 100 pour le muscle grand pectoral. Les muscles grands pectoraux ainsi que le gras sous-cutané et la peau qui les recouvrent représentent au total 14,6 p. 100 du poids vif. L'ensemble cuisses + pilons + reste de carcasse + cou, mais sans les ailes ni le croupion, représente $40 \mathrm{p} .100$ du poids vif dont près de 11 p. 100 pour la peau et le gras sous-cutané.

\section{TABLEAU 2}

Moyenne et écart-type des composantes des principales parties de la carcasse exprimée en p. 100 du poids de chaque partie.

Means and standard deviations of dissected components of a given carcass cut expressed as percent of each particular cut.

\begin{tabular}{|c|c|c|}
\hline $\begin{array}{l}\text { Elément disséqué } \\
\text { Cut }\end{array}$ & $\begin{array}{l}\text { Moyenne } \\
\text { Mean }\end{array}$ & $\begin{array}{c}\text { Ecart-type } \\
\text { Standard-deviation }\end{array}$ \\
\hline \multicolumn{3}{|l|}{$\begin{array}{l}\text { 1. Poitrine sans os (poids moyen : } 344,6 \mathrm{~g} \text { ) } \\
\text { Breast without bone (mean weight) }\end{array}$} \\
\hline Peau/Skin $\ldots \ldots \ldots \ldots \ldots \ldots$ & 21,7 & 2,00 \\
\hline Gras sous-cutané/Subcutaneous fat . . . . . . . . . & 2,3 & 0,75 \\
\hline Peau + gras sous-cutané $/ S k i n+S$. fat $\ldots \ldots \ldots$ & 24,1 & 2,54 \\
\hline Muscle grand pectoral/Pectolaris major muscle . . . . & 66,2 & 2,42 \\
\hline Muscles petits pectoraux/Pectolaris minor muscles . . & 9,2 & 0,65 \\
\hline \multicolumn{3}{|l|}{$\begin{array}{l}\text { 2. Cuisses + pilons (poids moyen : } 370,6 \mathrm{~g} \text { ) } \\
\text { Thighs + drumsticks }\end{array}$} \\
\hline Peau $\ldots \ldots \ldots \ldots \ldots \ldots \ldots \ldots$ & 17,6 & 1,67 \\
\hline Gras sous-cutané $\ldots \ldots \ldots \ldots \ldots \ldots \ldots$ & 12,0 & 2,99 \\
\hline Peau + gras sous-cutané $\ldots \ldots \ldots \ldots \ldots$ & 29,6 & 3,58 \\
\hline Autres tissus ${ }^{(1)} /$ Other tissues $\ldots \ldots \ldots \ldots$ & 70,4 & - \\
\hline \multicolumn{3}{|l|}{$\begin{array}{l}\text { 3. Reste de carcasse }+ \text { cou (poids moyen : } 396,2 \mathrm{~g} \text { ) } \\
\text { Remaining part }+ \text { neck }\end{array}$} \\
\hline Peau $\ldots \ldots \ldots \ldots \ldots \ldots \ldots \ldots$ & 22,1 & 1,89 \\
\hline Gras sous-cutané & 8,2 & 1,37 \\
\hline Peau + gras sous-cutané $\ldots \ldots \ldots \ldots$ & 30,3 & 2,65 \\
\hline Autres tissus $(1) \ldots \ldots \ldots \ldots \ldots \ldots \ldots$ & 69,7 & - \\
\hline \multicolumn{3}{|l|}{$\begin{array}{l}\text { 4. Total disséqué (2) (poids moyen : } 1111,4 \mathrm{~g} \text { ) } \\
\text { Total dissected }\end{array}$} \\
\hline 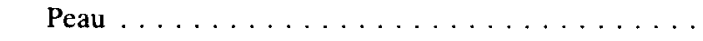 & 20,5 & 1,42 \\
\hline Gras sous-cutané $\ldots \ldots \ldots \ldots \ldots \ldots \ldots$ & 7,6 & 1,34 \\
\hline Peau + gras sous-cutané $\ldots \ldots \ldots \ldots \ldots$ & 28,1 & 2,30 \\
\hline Autres tissus $^{(1)} \ldots \ldots \ldots \ldots \ldots \ldots \ldots \ldots \ldots$ & 71,9 & - \\
\hline
\end{tabular}

(1) La part des autres tissus a été estimée par différence. Ils représentent les muscles, le tissu gras entre les muscles, les os et les tendons.

The part of other tissus was estimated by difference. They represent muscles, fatty tissue between muscles, bones and tendons.

(2) Le « total disséqué " correspond à la carcasse éviscérée avec le cou mais sans les ailes ni le croupion. "Total dissected" correponds to the eviscerated carcass with neck, but without wings and tail. 
Le tableau 2 indique les paramètres statistiques des éléments disséqués exprimés en p. 100 de chaque partie de carcasse. Dans notre échantillon, la peau et le gras souscutané constituent 24 p. 100 du poids total de la poitrine (sans os), le muscle grand pectoral 66 p. 100 et les petits pectoraux 9 p. 100 . Pour les autres parties de la carcasse, la peau et le gras sous-cutané représentent environ 30 p. 100 de l'ensemble.

Les corrélations calculées entre poids bruts des éléments entre eux, d'une part, et les corrélations entre rapports de chaque élément au poids vif entre eux, d'autre part, sont regroupées dans le tableau 3. On peut observer que les corrélations entre poids bruts sont généralement élevées et significativement positives, à l'exception de quelques unes faisant intervenir les abats consommables, le cou, le croupion ainsi que le gras sous-cutané de la poitrine. Le poids vif est corrélé positivement aux pourcentages du gras abdominal $(0,57)$, du gras sous-cutané $(0,3$ à 0,6$)$, et, de façon généralement non significative, avec les pourcentages de peau $(0,1$ à 0,5$)$. Entre les rapports élément de carcasse/poids vif, les corrélations sont souvent non significatives. La corrélation entre le pourcentage total de peau disséquée et le pourcentage total de gras sous-cutané dépasse légèrement le seuil de signification $(0,45)$.

Dans le tableau 4, sont présentées les corrélations entre le poids vif, le rapport gras abdominal/poids vif et les mesures de peau et de gras sous-cutané exprimées en p. 100 de la partie de carcasse correspondante. Les valeurs obtenues s'écartent peu de celles observées entre les mêmes variables exprimées en p. 100 du poids vif. Dans ce tableau, nous avons ajouté les variables composites peau + gras sous-cutané (variables numérotées 110 à 113), lesquelles sont moyennement corrélées avec le poids vif $(0,5$ à $0,6)$. On peut observer, par ailleurs, que l'ensemble cuisses + pilons constitue une bonne image des résultats de dissection du gras sous-cutané et de la peau de la carcasse entière : la corrélation est de 0,93 pour le pourcentage de tissus gras sous-cutané, de 0,72 pour le pourcentage de peau et de 0,86 pour le pourcentage global peau + gras sous-cutané. La forte valeur observée pour le gras sous-cutané n'est pas étonnante puisque le gras sous-cutané des cuisses et pilons représente la moitié du total mesuré.

\section{Discussion et conclusion}

Les résultats observés au cours de la présente expérience ne concernent qu'un échantillon limité de canards : une seule espèce, une seule origine commerciale, un seul sexe et un seul âge d'abattage. Il n'est donc pas possible de les généraliser à tous les échantillons de canards (sexe, âge, type génétique, stade physiologique) qu'on peut trouver sur le marché. Ils permettent néanmoins d'avoir une idée sur la variabilité phénotypique intra-échantillon et peuvent servir de point de repère avant d'étudier tel ou tel facteur de variation. En particulier, les corrélations avec le poids vif suggèrent qu'une augmentation de la vitesse de croissance devrait s'accompagner de dépôts gras plus importants, d'une diminution de la part relative des abats, du cou et des ailes, et d'une variation faible et non significative du pourcentage de la carcasse éviscérée et de la peau. Comme dans notre travail précédent (RICARD, 1986), la variabilité des tissus gras est très forte: les coefficients de variation varient de 20 à $30 \mathrm{p}$. 100. C'est également le cas chez le poulet (RICARD, 1984), où on sait que ce type de tissu varie fortement en fonction du génotype et de l'environnement. On doit donc pouvoir, chez le canard comme chez le poulet, obtenir des carcasses maigres soit par sélection, soit en utilisant différentes techniques d'élevage. L'un de nos objectifs était de caractériser la 


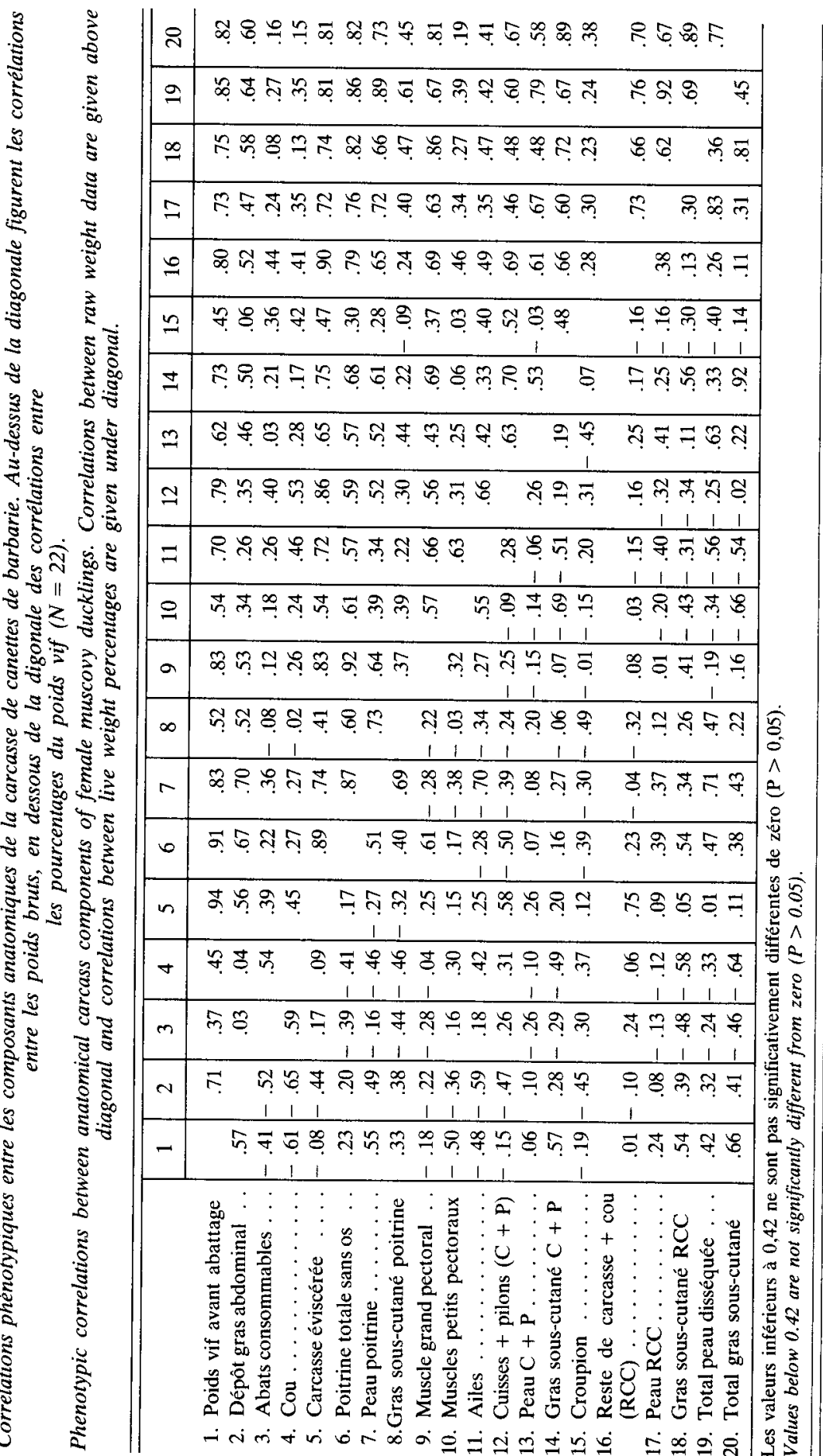




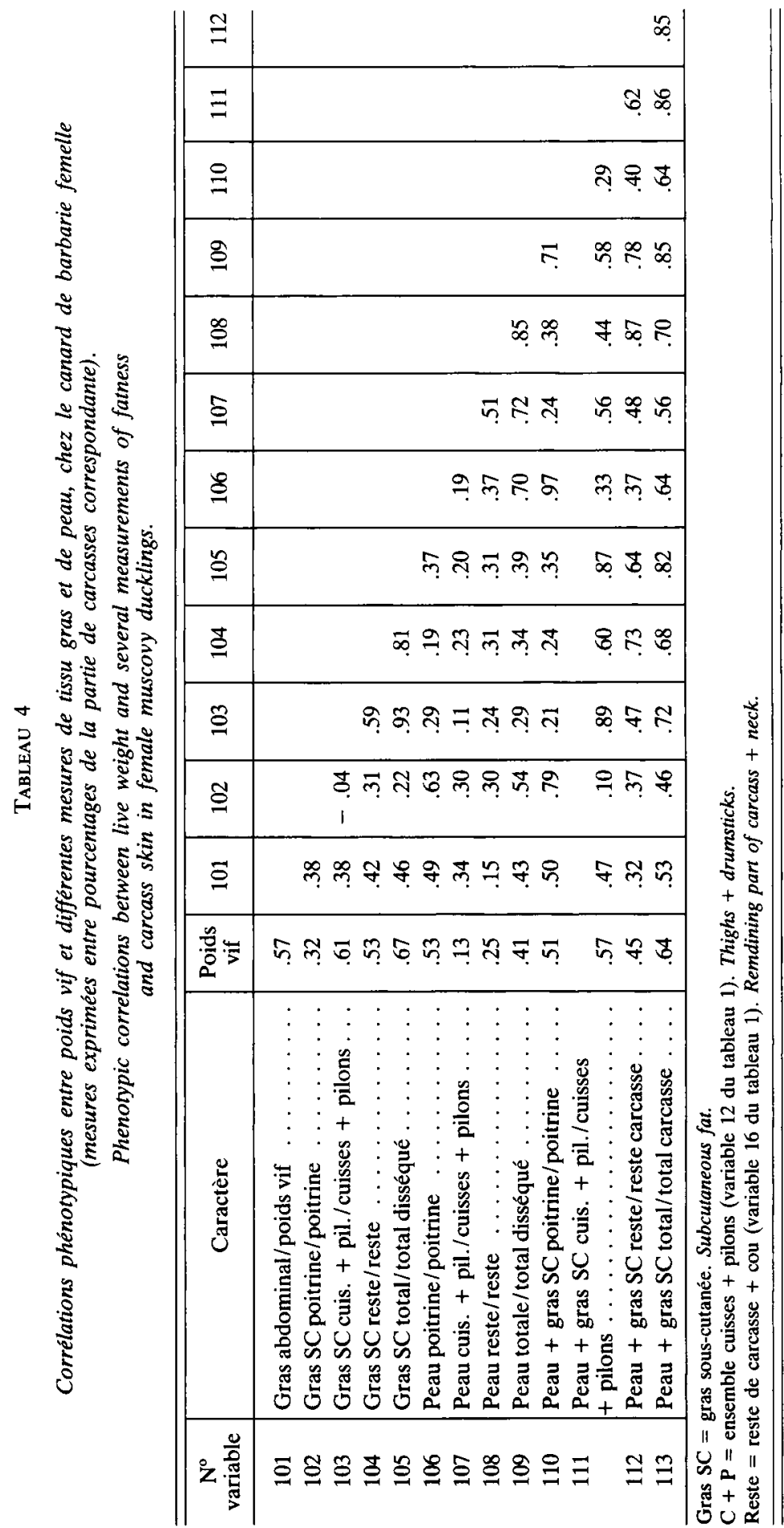




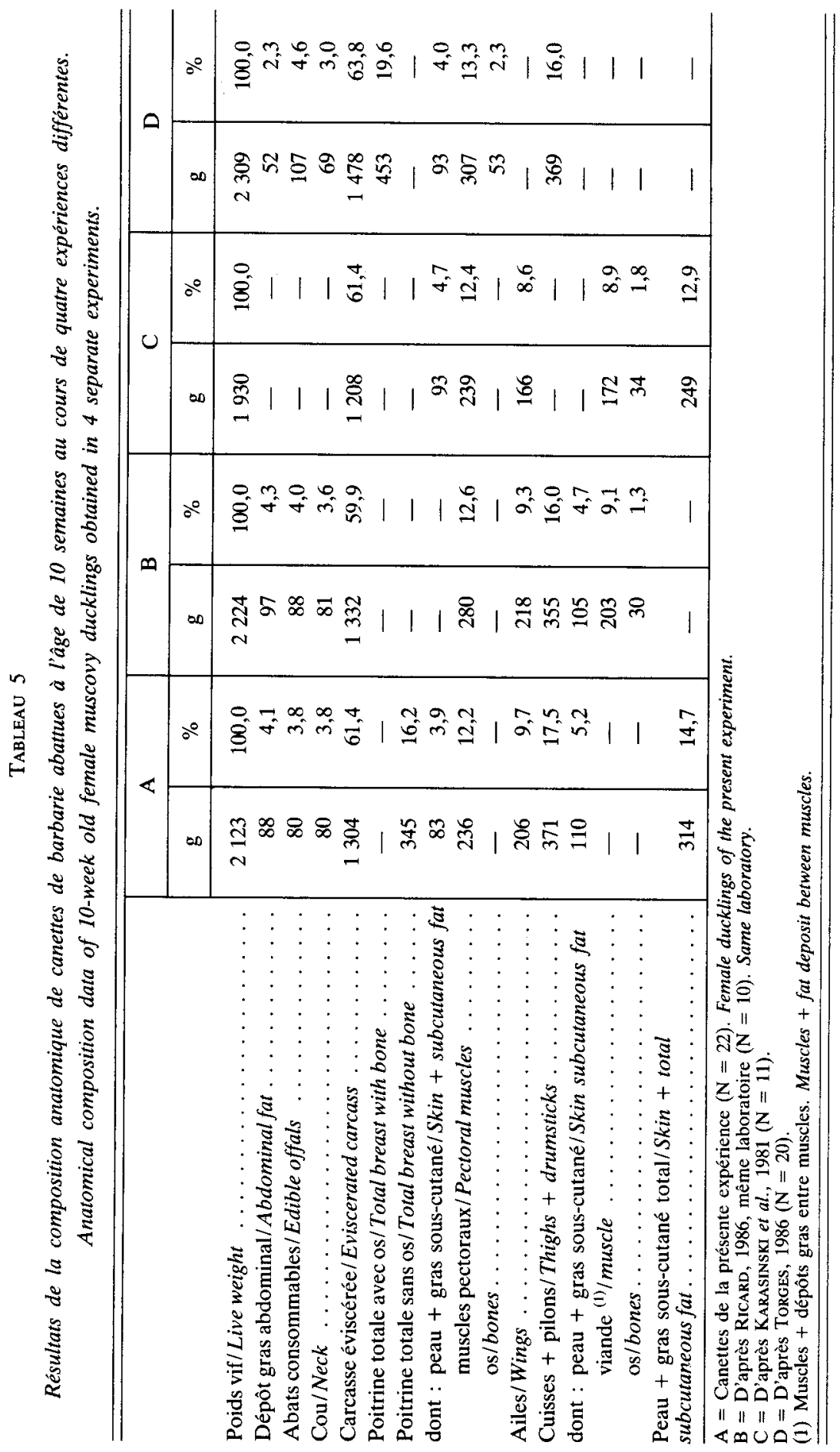


carcasse des canettes de barbarie en vue de préciser son intérêt pour la découpe. Nous nous sommes donc limités à une dissection anatomique et nous n'avons pas de données relatives à l'analyse chimique de ces carcasses, en particulier de leur teneur en lipides. Une telle analyse s'avérerait nécessaire si on désirait préciser la qualité diététique des carcasses de canard.

Nous avons cité plusieurs références de travaux concernant la composition anatomique du canard de barbarie. Dans le tableau 5, sont rassemblés les résultats qui concernent également des femelles abattues à l'âge de 10 semaines : une expérience antérieure réalisée dans notre propre laboratoire (RICARD, 1986), une expérience polonaise (KARASINSKI et al., 1981) et une expérience allemande (Torges, 1986). Le poids vif moyen des animaux de notre précédente expérience était un peu supérieur à celui du présent échantillon, ce qu'on peut attribuer à une variation liée à l'erreur d'échantillonnage. Les différences de croissance observées dans les travaux de KARASINSKI et de TORGES sont probablement liées à des conditions expérimentales non identiques pour beaucoup de facteurs, en particulier la souche. Par ailleurs, les proportions des différents éléments de la carcasse, exprimées en p. 100 du poids vif, sont voisines dans les quatre séries de résultats, les écarts étant probablement dus à de légères variations dans les techniques de dissection. C'est le cas, par exemple, du pourcentage du gras abdominal, plus faible chez les animaux de ToRGes que chez les nôtres : il faudrait bien connaître la technique utilisée dans les deux cas avant de conclure à une différence. La comparaison des deux séries de mesures obtenues dans notre laboratoire (lots $\mathrm{A}$ et $\mathrm{B}$ du tableau 5), fait apparaître une différence dans le rendement éviscéré, laquelle est due à un plus fort pourcentage de l'ensemble cuisses+ pilons dans le lot A (17,5 p. 100) que dans le lot B (16,0 p. 100). Cette différence peut s'expliquer par les aléas de l'échantillonnage. Mais on peut aussi remarquer que le poids brut des cuisses et pilons varie peu entre les lots A et B (371 et $355 \mathrm{~g}$ ), comparé à des vitesses de croissance différentes (poids vif de 2123 et $2224 \mathrm{~g}$, respectivement). Or, il a été observé précédemment que le poids des cuisses et pilons variait peu après l'âge de 8 semaines (RICARD, 1986) et qu'il pouvait même diminuer malgré une augmentation du poids vif, du moins chez les mâles gavés (BABILE et al., 1986).

Une autre comparaison intéressante peut être faite avec les poulets d'un croisement standard de type Cornish $\times$ White Rock étudiés précédemment dans notre laboratoire (RICARD, 1984, lot A) : la canette de barbarie a un pourcentage de gras abdominal, par rapport au poids vif, plus élevé $(4,1$ p. 100$)$ que ces poulets $(2,8$ p. 100$)$; elle présente également un plus fort pourcentage de peau $(10,7$ contre 6,0$)$ et de gras sous-cutané $(4,0$ contre 3,2$)$. C'est probablement la plus grande importance de l'ensemble peau+ gras sous-cutané qui fait considérer le canard comme une volaille plus " grasse » que le poulet. Mais il faut dire que le stade physiologique des deux groupes d'animaux n'était pas le même : les poulets (mâles) étaient abattus à un poids vif de $2 \mathrm{~kg}$ alors que le poids adulte des coqs de souches chair peut dépasser $5 \mathrm{~kg}$; les canards de la présente expérience (femelles) étaient également abattus à un poids vif voisin de $2 \mathrm{~kg}$, mais le poids adulte des canes de barbarie dépasse rarement $3 \mathrm{~kg}$ (de Carville, communication personnelle).

Les corrélations peuvent également être comparées entre les espèces canard et poulet. Dans le tableau 6, nous avons rassemblé les corrélations entre éléments de tissu gras et de peau chez les coquelets du croisement Cornish $\times$ White Rock précité (RICARD, 1984) et celles obtenues sur les canettes de barbarie de la présente expérience. On constate que le pourcentage de gras abdominal est plus fortement corrélé avec celui de gras sous-cutané chez le poulet que chez le canard. Dans les deux cas, le gras sous- 


\section{TABleau 6}

Comparaison de quelques corrélations observées chez le poulet et chez les canettes de barbarie de la présente expérience.

Comparison between correlations obtained in broiler chickens and those obtained in female muscovy ducklings in the present experiment.

\begin{tabular}{|c|c|c|}
\hline $\begin{array}{l}\text { Variables corrélées }{ }^{(1)} \\
\text { Correlated variables }\end{array}$ & $\begin{array}{l}\text { Poulets } \\
\text { Chickens }\end{array}$ & $\begin{array}{l}\text { Canards }{ }^{(3)} \\
\text { Ducks }\end{array}$ \\
\hline $\begin{array}{l}\% \text { Gras abdo. }-\% \text { Gras SC cuisses + pilons } \ldots \ldots \\
\text { Abdominal fat }- \text { Subcutaneous fat, thighs }+ \text { drumsticks }\end{array}$ & 0,46 & 0,28 \\
\hline $\begin{array}{l}\% \text { Gras abdo. }-\% \text { Gras SC total } \ldots \ldots \ldots \ldots \\
\text { Abdominal fat }- \text { Total SC fat }\end{array}$ & 0,57 & 0,41 \\
\hline $\begin{array}{l}\% \text { Gras abdo. }-\% \text { Peau cuisses }+ \text { pilons } \ldots \ldots \\
\text { Abdominal fat }- \text { Skin, thighs }+ \text { drumsticks }\end{array}$ & 0,30 & 0,10 \\
\hline $\begin{array}{l}\% \text { Gras abdo. }-\% \text { Peau totale } \ldots \ldots \ldots \ldots \\
\text { Abdominal fat }- \text { Total skin }\end{array}$ & 0,33 & 0,32 \\
\hline$\%$ Gras SC C $+\mathrm{P}-\%$ Gras SC total $\ldots \ldots \ldots$ & 0,92 & 0,92 \\
\hline$\%$ Gras $\mathrm{SCC}+\mathrm{P}-\%$ Peau $\mathrm{C}+\mathrm{P}$ & 0,42 & 0,19 \\
\hline$\%$ Peau, cuisses + pilons $-\%$ Peau totale $\ldots \ldots$ & 0,95 & 0,63 \\
\hline$\%$ Gras SC total $-\%$ Peau totale $\ldots \ldots \ldots \ldots$ & 0,25 & 0,45 \\
\hline
\end{tabular}

(1) Corrélations entre les pourcentages rapportés au poids vif. Correlations between percentages related to live weight.

(2) Les corrélations concernant le poulet sont celles publiées par RICARD (1984) pour le croisement Cornish $\times$ White Rock $(\mathrm{N}=16$ coquelets $)$.

Correlations reported by RICARD (1984) in chickens of Cornish $\times$ white Rock crossing ( $N=16$ chickens).

(3) Canettes de barbarie de la présente expérience $(\mathrm{N}=22)$. Female muscovy ducklings of the present experiment.

cutané et la peau des cuisses et pilons sont fortement corrélés aux pourcentages de gras sous-cutané total et de peau totale disséqués. Par ailleurs, les corrélations entre pourcentages de gras sous-cutané et de peau sont relativement faibles dans les deux échantillons. On peut donc penser que dans les deux cas l'ensemble cuisses + pilons peut fournir une bonne image de la composition anatomique de la carcasse totale et que peau et gras sous-cutané ont un développement relativement indépendant l'un de l'autre. Il serait intéressant d'avoir des données sur d'autres espèces pour voir si on peut généraliser ces conclusions à l'ensemble des volailles.

Reçu en novembre 1986.

Accepté en mars 1987.

\section{Remerciements}

Les carcasses faisant l'objet de la présente expérience provenaient d'une expérimentation zootechnique (non publiée), conduite à la Station de Recherches avicoles par $\mathrm{H}$. de CARville. Ce dernier, ainsi que Thérèse GifFard-Lescor ont participé aux travaux de dissection. Nous les en remercions vivement. 


\section{Summary \\ Anatomical characteristics of female muscovy duckling carcasses}

A group of 22 females muscovy ducklings was slaughtered at 10 weeks of age to assess the share of different carcass components, especially fat deposit and skin, and to estimate correlations between them.

Weight and percentage of each carcass component are given in tables 1 and 2, correlations in tables 3 and 4 . Breast (without bone), wings, thighs + drumsticks, abdominal fat, total dissected subcutaneous fat and total dissected skin represented $16.2,9.7,17.5,4.1,4.0$ and 10.7 percent of live weight, respectively. Correlations between raw weights of the different components were generally high. Between live weight and fat deposit percentages, correlations of about .6 were obtained. As far as relationships between live weight percentages are concerned, skin and subcutaneous fat developments appeared relatively independant.

Results were compared with 3 other experiments involving 10-week old female muscovy carcasses. These comparisons are summarized in table 5 and the same general conclusion were drawn. As shown in table 6, thighs + drumsticks gave a good estimation for total skin and subcutaneous fat percentages of the whole carcass, which confirmed results obtained in broiler chickens.

Our results may be of interest for the cutting of female muscovy ducklings. tions.

Key words : Muscovy ducklings, carcass parts, abdominal fat, subcutaneous fat, skin, correla-

\section{Références bibliographiques}

Babile R., Auvergne A., Delpech P., 1986. Croissance et découpe commerciale de canards mâles de barbarie. Effet du type génétique, de la conduite alimentaire et de l'âge. C.R. $7^{\mathfrak{e}}$ Conf. Europ. Avic., Paris, Ed. LARbIER M., 828-832.

Chamberlain F.W., 1943. Atlas of avian anatomy. Michigan Agric. Exp. Sta., Memoir. Bull. 5.

KaRasinski D., Domanski J., KolodziEJ H., 1981. Results of dissection of 10-week old ducks of breeds Cairina moschata. Pekin, Orpington and hybrids. Rocz. Akad. Roln. Poznan, 29, 9199.

Pingel H., Schneider K.H., 1981. Effect of age and sex on meat yield and carcass composition of ducks, geese and muscovy ducks. In "Quality of Poultry Meat ", ed. Mulder R.W., ScheEle C.W. \& Veerkamp C.H., Beekbergen, Pays-Bas, 28-37 (Proc. $5^{\text {th }}$ Europ. Symp. on Quality of Poultry Meat).

RiCARD F.H., 1984. Relations entre différents dépôts gras et éléments de peau chez le poulet. Ann. Zootech., 33, 149-160.

RICARD F.H., 1986. Composition anatomique de la carcasse du canard mulard comparée aux deux types parentaux. C.R. Conférence Avicole W.P.S.A.-SIMAVIP, cahier $\mathrm{n}^{\circ}$ 3, 47-65. Ed. Groupe Français de la W.P.S.A., Nouzilly, France.

Rомвоц I., 1980. Evoluzione della composizione della carcassa nell'anatra muschiata. Zoot. Nutr. Anim., 6, 319-330.

Seemann G., 1983. Yield and cutling of Pekin and Muscovy ducks. In "Qualité des Viandes de Volailles ", Ed. Lahellec C., Ricard F.H. \& Colin P., Ploufragan, France, 352-360 (C.R. $6^{\circ}$ Symp. Europ. sur la Qualité des Viandes de Volailles).

Torges H.G., 1986. Ausschachtungsergebnisse von moschusenten in Abhängigkeit vom alter und geschlecht. Arch. Geflügelk., 50, 31-41.

Ziegler W., Petersen J., Tuller R., 1985. Einfluss von alter und fütterungsintensität zuf wachstum und schlachtkörperbeschaffenheit von moschuserpeln, Teil 1. Arch. Geflügelk., 49, 98-107. 